\title{
Free vibration of sandwich plates and shells by using Zig-Zag function
}

\author{
S. Brischetto ${ }^{\mathrm{a}, *}$, E. Carrera ${ }^{\mathrm{a}}$ and L. Demasi ${ }^{\mathrm{b}}$ \\ ${ }^{a}$ Department of Aeronautics and Space Engineering, Politecnico di Torino, Torino, Italy \\ ${ }^{\mathrm{b}}$ Department of Aerospace Engineering, San Diego State University, San Diego, CA, USA
}

Received 30 June 2008

Revised September 2008

\begin{abstract}
This paper analyses the free vibration response of sandwich curved and flat panels by introducing the zig-zag function $(-1)^{k} \zeta_{k}$ (ZZF) in the displacement models of classical and higher order two-dimensional shell theories. The main advantage of ZZF is the introduction of a discontinuity in the first derivative, zig-zag effect, of the displacements distribution with correspondence to the core/faces interfaces. Results including and discarding ZZF are compared. Several values of face-to-core stiffness ratio (FCSR) and geometrical plate/shell parameters have been analyzed. Both fundamental vibration modes and those corresponding to high wave numbers are considered in the analysis. It is concluded that: (1) ZZF is highly recommended in the free vibration analysis of sandwich plates and shells; (2) the use of ZZF makes the error almost independent by FCSR parameter; (3) ZZF is easy to implement and its use should be preferred with respect to other 'more cumbersome' refined theories.
\end{abstract}

\section{Introduction}

Nowadays sandwich structures are used to build large portions of advanced structural elements for aerospace, automotive and ship vehicles. Typical sandwich structures are constituted by assembling of flat and/or curved panels. The analysis and design of these structures involve various topics. An accurate evaluation of the vibration response is one of the key-point for a rational use of sandwich structures [16].

As significant features sandwich structures are characterized by a soft core between two stiffer faces. They consist of a three layered structure. The discontinuity of mechanical properties between faces and core introduces a discontinuity of deformed core-faces planes at the interfaces, see Fig. 1. This is also known as Zig-Zag effect in laminated structures [5]. These discontinuities make difficult the use of classical theories such as Kirchhoff [15] or Reissner-Mindlin [20] type theories. In order to trace an accurate vibration response of sandwich structures see the books by Zenkert [25], Bitzer [2] and Vinson [24]. So called layer-wise models, in which the three layers are treated as three independent layers, can be used to capture the above ZZ form, see the overviews by Burton and Noor [4], Noor, Burton and Bert [19], Altenbach [1], Librescu and Hause [17], Vinson [23], Carrera and Brischetto [8], Demasi [13]. These models could result computational expensive.

In the framework of mixed multilayered plate theories, Murakami [18] proposed a Zig-Zag function ZZF able to reproduce the described slope discontinuity. Equivalent single layer models (the three layers are treated as one-layer equivalent plate) with only displacement unknowns can be developed on the basis of ZZF. The advantages of using the ZZF to analyse the multilayered anisotropic plates and shells as well as the Finite Element implementation have been discussed by Carrera [6] and Demasi [12], respectively. Recent papers have shown the effectiveness of ZZF in the bending analyses of sandwich plates with soft core [3,13]. The obtained results have encouraged the present analysis which is direct to explore the effectiveness of ZZF in the free vibration response of sandwich plates and shells. Various values of geometrical parameters (length, thickness, radius) are considered along with several face-to-core stiffness ratios to highlight the capability of ZZF.

*Corresponding author: Salvatore Brischetto, Department of Aeronautics and Space Engineering, Politecnico di Torino, Corso Duca degli Abruzzi 24, 10129, Torino, Italy. Tel.: +39 011564 6869; Fax: +39 011564 6899; E-mail: salvatore.brischetto@polito.it. 

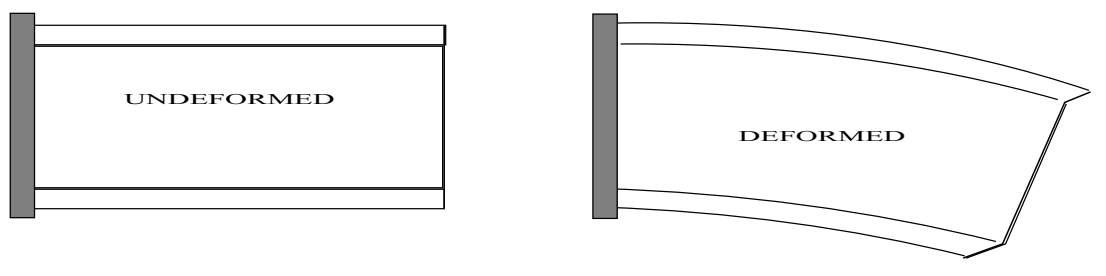

Fig. 1. Undeformed and deformed plane of a sandwich structure.
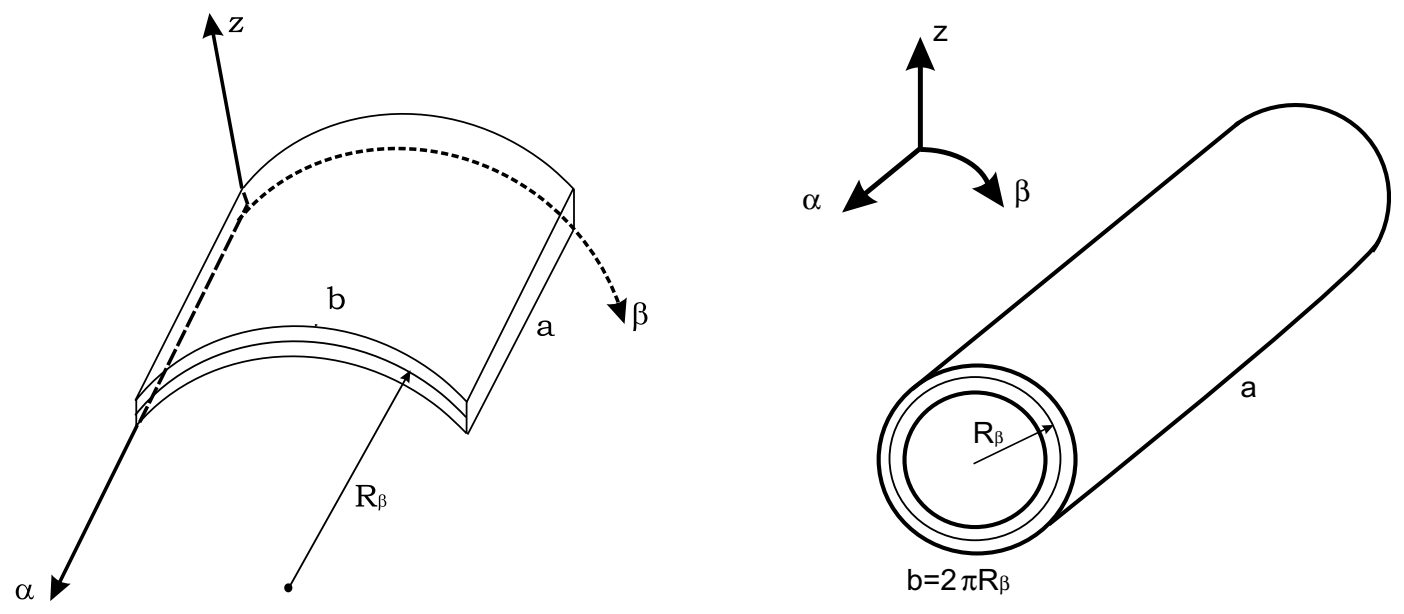

Fig. 2. Geometry and notations for the Ren (left) and the Varadan and Baskar (right) shell.

\section{The Zig-Zag function}

A sandwich plate/shell composed by 3 layers, perfectly bonded together, is considered. $z$ is the thickness coordinate of the whole structure, $z_{k}$ is the layer thickness coordinate. $h$ is the plate/shell thickness. The not dimensioned layer coordinate $\zeta_{k}=\left(2 z_{k}\right) / h_{k}$ is further introduced ( $h_{k}$ is the thickness of the $k^{t h}$ layer). $\alpha$ and $\beta$ are the shell orthogonal curvilinear coordinates over the reference surface $\Omega$. Plates have orthogonal rectilinear coordinates $(x, y, z)$. Results will be restricted to cylindrical shell geometries and the correspondent radius of curvature will be denoted by $R_{\beta}$, see Fig. 2.

Murakami's Zig-Zag Function $Z(z)$ was defined according to the following formula [18]:

$$
Z(z)=(-1)^{k} \zeta_{k} .
$$

$Z(z)$ has the following properties:

1. it is piece-wise linear function of the layer coordinates $z_{k}$;

2. $Z(z)$ has unit amplitude for the whole layers;

3. the slope $Z^{\prime}(z)=\frac{d Z}{d z}$ assumes opposite sign between two-adjacent layers. Its amplitude is layer thickness independent.

A plot of $Z(z)$ is given in Fig. 3. ZZF can be used in displacement $u(z)$ to introduce discontinuous slopes with correspondence to layer interfaces. If a linear polynomial is considered, in order to expand the displacement along the thickness, one has:

$$
u(z)=c^{0}+c^{1} z,
$$

where $c^{0}, c^{1}$ are the amplitudes of the uniform and linear terms of $u(z)$, respectively. By adding ZZF one has:

$$
u(z)=c^{0}+c^{1} z+c^{Z} Z(z),
$$



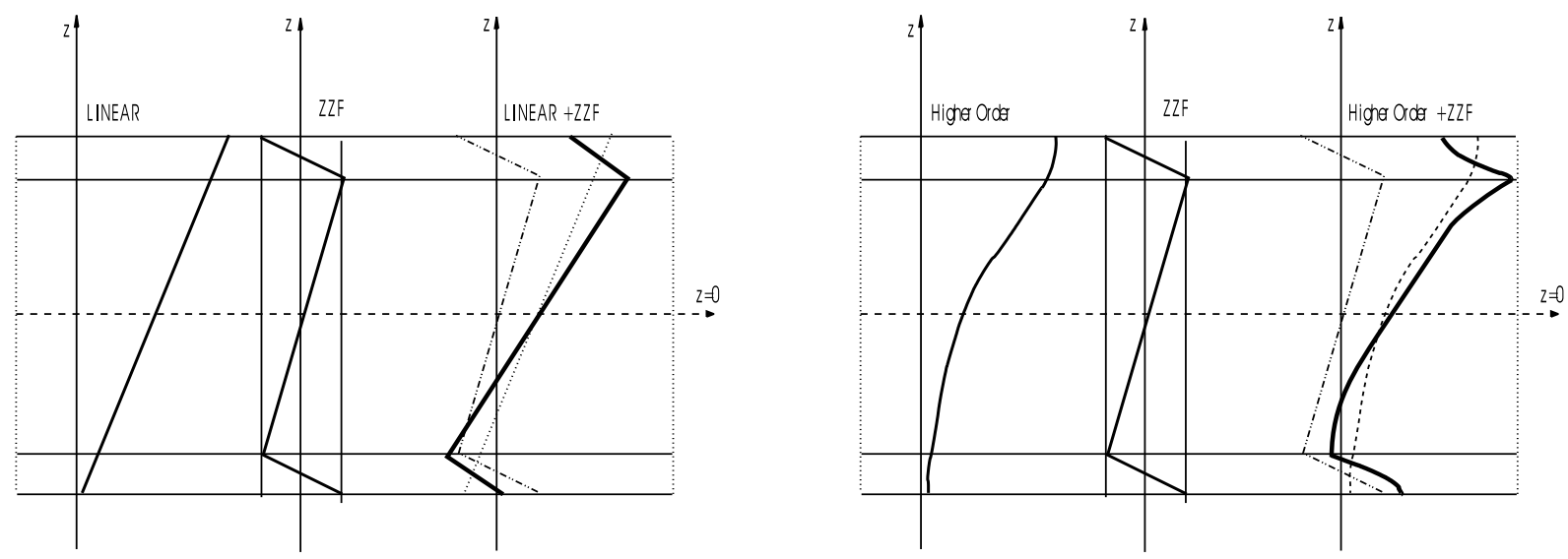

Fig. 3. Effect of adding the Zig-Zag function: linear case (left) and higher order case (right).

$c^{Z}$ is the amplitude of ZZF. Displacements with and without ZZF are compared in Fig. 3, which makes evident how $Z(z)$ emulates $\mathrm{ZZ}$ effects, that are very relevant in sandwich structures. $Z(z)$ can be also used in conjunction to higher $N$-order expansions (see Fig. 3):

$$
u(z)=c^{0}+c^{1} z+c^{2} z^{2}+\ldots+c^{N-1} z^{N-1}+c^{N} z^{N}+c^{Z} Z(z) .
$$

\section{Considered theories}

Classical theories for sandwich shells, such Kirchhoff (Classical Lamination Theory, CLT), Reissner-Mindlin (First order Shear Deformation Theory, FSDT) and Higher order Shear Deformation Theory (HSDT), see the review papers by Librescu and Hause [17] and Vinson [23], do not account for ZZ effect. A possible use of ZZF would consist to enhance classical models by 'simply' adding $Z(z)$ in their displacement fields. Let consider a linear distribution of a displacement component in the thickness direction $z$, as it is in CLT and FSDT:

$$
u(\alpha, \beta, z)=u^{0}(\alpha, \beta)+z u^{1}(\alpha, \beta)
$$

where $u(\alpha, \beta, z)$ is the displacement component along the $\alpha$ or $\beta$ direction of the generic point $P$ in a given Cartesian reference system; $u^{0}(\alpha, \beta)$ is the value of $u$ with correspondence to the reference surface $\Omega$ to which correspondence one has $z=0 ; u^{1}(\alpha, \beta)$ is an additional variable ( $u^{1}$ has the geometrical meaning of rotation of the normal to $\Omega$ in $P$ ). If FSDT applications are considered, Eq. (5) is retained only for the in-plane components $\left(u_{1}\right.$ coincides to the derivative of the transverse displacement with respect to the in-plane coordinates in the CLT case). ZZF offers a possibility to introduce $\mathrm{ZZ}$ effect in Eq. (5), in fact:

$$
u(\alpha, \beta, z)=u^{0}(\alpha, \beta)+z u^{1}(\alpha, \beta)+(-1)^{k} \zeta_{k} u^{Z}(\alpha, \beta) .
$$

The following remarks can be made:

1. the additional degree of freedom $u^{Z}$ has a meaning of displacement;

2. the amplitude $u^{Z}$ is layer independent: $u^{Z}$ has an intrinsic equivalent single layer description. At a first glance this fact could appear as a strong limitation of ZZF. Actually ZZF does not differ from other Zig-Zag theories, such as Ambartsumian Multilayered Theories and Lekhnitskii Multilayered Theories, as it has been detailed in [5];

3. ZZF can be used for both in-plane and out-of-plane displacement components. 
Table 1

Number of degrees of freedom for the considered sandwich plate/shell theories

\begin{tabular}{lr}
\hline Theory & d.o.f. \\
\hline CLT & 3 \\
FSDT & 5 \\
ED1 & 6 \\
EDZ1 & 9 \\
ED4 & 15 \\
EDZ3 & 15 \\
\hline
\end{tabular}

\subsection{Refinements of FSDT}

The applications of ZZF to FSDT lead to the following displacement model:

$$
\begin{aligned}
& u_{1}(\alpha, \beta, z)=u_{1}^{0}(\alpha, \beta)+z u_{1}^{1}(\alpha, \beta)+(-1)^{k} \zeta_{k} u_{1}^{Z}(\alpha, \beta) \\
& u_{2}(\alpha, \beta, z)=u_{2}^{0}(\alpha, \beta)+z u_{2}^{1}(\alpha, \beta)+(-1)^{k} \zeta_{k} u_{2}^{Z}(\alpha, \beta) \\
& u_{3}(\alpha, \beta, z)=u_{3}^{0}(\alpha, \beta)
\end{aligned}
$$

Subscripts 1, 2 and 3 denote displacement components in the three orthogonal directions of a given plate/shell reference system. The third one refers to the thickness-transverse $z$-direction. The enhanced FSDT model has seven degrees of freedom, while the classical FSDT one has only five.

\subsection{Refinement of FSDT by inclusion of ZZ effects and transverse normal strains $\epsilon_{z z}$}

The displacement model which includes transverse normal strains as well as ZZ effect in FSDT is:

$$
\begin{aligned}
& u_{1}(\alpha, \beta, z)=u_{1}^{0}(\alpha, \beta)+z u_{1}^{1}(\alpha, \beta)+(-1)^{k} \zeta_{k} u_{1}^{Z}(\alpha, \beta) \\
& u_{2}(\alpha, \beta, z)=u_{2}^{0}(\alpha, \beta)+z u_{2}^{1}(\alpha, \beta)+(-1)^{k} \zeta_{k} u_{2}^{Z}(\alpha, \beta) \\
& u_{3}(\alpha, \beta, z)=u_{3}^{0}(\alpha, \beta)+z u_{3}^{1}(\alpha, \beta)+(-1)^{k} \zeta_{k} u_{3}^{Z}(\alpha, \beta)
\end{aligned}
$$

The related theories are denoted as ED1 and EDZ1 depending on the inclusion or not of the ZZF, respectively. The letter E means Equivalent Single Layer theory, D states for Displacements formulation, the number indicates the order of expansion in $z$ direction (form linear (1) to fourth (4)). The adding of $Z$ means inclusion of zig-zag function.

\subsection{Higher order theories with ZZ function}

ZZF can be used to introduce ZZ effect in any HSDT type expansions. The expansions considered in this work make use of power of $z$ polynomials:

$$
\begin{aligned}
u_{i}(\alpha, \beta, z)= & u_{i}^{0}(\alpha, \beta)+z u_{i}^{1}(\alpha, \beta)+z^{2} u_{i}^{2}(\alpha, \beta)+\ldots+z^{N} u_{i}^{N}(\alpha, \beta)+(-1)^{k} \zeta_{k} u_{i}^{Z}(\alpha, \beta), \\
& i=1,2,3 .
\end{aligned}
$$

$N$ is the order of expansion. The cases $N=1,2,3,4$ will be considered in the numerical discussion. The application will refer to the cases of ED4 and EDZ3 theories that correspond to $N=4$ and $N=3$, respectively (the latter includes ZZF). A summary of degrees of freedom for the considered theories is given in Table 1. It appears clear that the computational cost of EDZ1 theory is much more less than ED4 theory. EDZ3 theory has the same number of degrees of freedom of the ED4. 
Table 2

Comparison of various theories to evaluate the fundamental frequency amplitude for the plate. $m=n=1 . \bar{\omega}=\omega \sqrt{\frac{a^{4}(\rho)_{\text {skin }}}{(E)_{\text {skin }} h^{2}}}$

\begin{tabular}{lcccccccc}
\hline$a / h$ & 4 & $E r r$. & 10 & Err. & 100 & Err. & 1000 & $E r r$. \\
\hline & & & & \multicolumn{2}{c}{$F C S R=10$} \\
$3 D$ & 5.0921 & $\%$ & 7.4092 & $\%$ & 8.3960 & $\%$ & 8.4150 & $\%$ \\
$C L T$ & 7.6822 & $(50.8)$ & 8.2782 & $(11.7)$ & 8.4070 & $(0.13)$ & 8.4083 & $(0.08)$ \\
$F S D T$ & 6.3942 & $(25.6)$ & 7.9276 & $(7.00)$ & 8.4030 & $(0.08)$ & 8.4083 & $(0.08)$ \\
$E D 1$ & 6.3942 & $(25.6)$ & 7.9276 & $(7.00)$ & 8.4030 & $(0.08)$ & 8.4083 & $(0.08)$ \\
$E D 4$ & 5.2422 & $(2.95)$ & 7.4801 & $(0.96)$ & 8.3970 & $(0.01)$ & 8.4082 & $(0.08)$ \\
$E D Z 1$ & 5.0607 & $(0.62)$ & 7.3860 & $(0.31)$ & 8.3956 & $(0.00)$ & 8.4082 & $(0.08)$ \\
$E D Z 3$ & 5.1149 & $(0.45)$ & 7.4110 & $(0.02)$ & 8.3960 & $(0.00)$ & 8.4082 & $(0.08)$ \\
& & & & $F C S R=10^{5}$ & & & \\
$3 D$ & 0.6093 & $\%$ & 0.6355 & $\%$ & 1.9864 & $\%$ & 8.5150 & $\%$ \\
$C L T$ & 8.4625 & $(>100)$ & 9.2802 & $(>100)$ & 9.4627 & $(>100)$ & 9.4646 & $(11.1)$ \\
$F S D T$ & 6.8462 & $(>100)$ & 8.7970 & $(>100)$ & 9.4570 & $(>100)$ & 9.4645 & $(11.1)$ \\
$E D 1$ & 6.8462 & $(>100)$ & 8.7970 & $(>100)$ & 9.4570 & $(>100)$ & 9.4645 & $(11.1)$ \\
$E D 4$ & 2.0922 & $(>100)$ & 4.5512 & $(>100)$ & 9.3079 & $(>100)$ & 9.4630 & $(11.1)$ \\
$E D Z 1$ & 0.6096 & $(0.04)$ & 0.6357 & $(0.03)$ & 1.9865 & $(0.00)$ & 8.5061 & $(0.10)$ \\
$E D Z 3$ & 0.7088 & $(16.3)$ & 0.7319 & $(15.2)$ & 2.0168 & $(1.53)$ & 8.5064 & $(0.10)$ \\
\hline
\end{tabular}

\section{Numerical results and discussion}

The various plate/shell models described above have been adopted in the framework of Carrera's Unified Formulation CUF [14] for plates and shells which has been detailed in previous works [7]. Closed form solutions are herein discussed for the case of simply supported sandwich plates/shells made of isotropic layers. Navier-type closed form solutions can be found by assuming the following harmonic forms for unknown displacements:

$$
\begin{aligned}
u_{1}^{\tau} & =\sum_{m, n} U_{1}^{\tau} \cos \frac{m \pi \alpha}{a} \sin \frac{n \pi \beta}{b} e^{i \omega_{m n} \hat{t}} \\
u_{2}^{\tau} & =\sum_{m, n} U_{2}^{\tau} \sin \frac{m \pi \alpha}{a} \cos \frac{n \pi \beta}{b} e^{i \omega_{m n} \hat{t}} \\
u_{3}^{\tau} & =\sum_{m, n} U_{3}^{\tau} \sin \frac{m \pi \alpha}{a} \sin \frac{n \pi \beta}{b} e^{i \omega_{m n} \hat{t}} \quad \tau=0,1,2,3,4, Z
\end{aligned}
$$

which correspond to simply-supported boundary conditions. $a_{k}$ and $b_{k}$ are the shell lengths in the $\alpha_{k}$ and $\beta_{k}$ directions, respectively; $m$ and $n$ are the correspondent wave numbers; $\hat{t}$ denotes time; $i=\sqrt{-1} ; \omega_{m n}$ is the circular frequency. The free vibration response leads to an eigenvalue problem. Details of related governing equations and solution procedures are herein omitted, but can be found in $[7,11]$.

Poisson's locking phenomena has been approached according to the findings in $[9,10]$. The attention has been focused to evaluate the effectiveness of $\mathrm{ZZ}$ function to improve the results accuracy in the case of vibration response of sandwich shells. These models are more efficient than classical and refined theories that do not make use of $\mathrm{ZZ}$ function. CLT, FSDT and higher order theories (ED1, ED4) are compared to theories that use ZZ functions (EDZ1, EDZ3). Results related to intermediate cases such as ED2, ED3 either EDZ2 have been omitted for sake of brevity. 3D solution has been provided via layer-wise mixed theories, see [7]. Degrees of freedom for the compared theories are given in Table 1, that permits to estimate the computational cost of each plate/shell theory.

Three different geometries have been considered: square plate; cylindrical panel; closed cylindrical shell. Various values of length-to-thickness ratio LTR $(a / h)$, and radius-to-thickness ratio RTR $\left(R_{\beta} / h\right)$ have been considered. The physical reason of Zig-Zag form for displacements field in the thickness direction is strongly due to the variation of mechanical properties between core and faces. A mechanical parameter FCSR (face-to-core-stiffness ratio) has been introduced to make evident such a discontinuity. Faces made of aluminum alloy (Al2024, $E=73000$ [MPa], $\nu=0.34$ ) with thickness $h_{f}=1[\mathrm{~mm}]$ have been considered. An isotropic foam core with thickness $h_{c}=8$ [mm] has been addressed $(\nu=0.34)$; the mechanical properties of the core have been varied from $10^{-1}$ to $10^{-5}$, that 
Table 3

Comparison of various theories to evaluate the fundamental frequency amplitude for the plate. $m=n=100 . \bar{\omega}=\omega \sqrt{\frac{a^{4}(\rho)_{\text {skin }}}{(E)_{\text {skin }} h^{2}}}$

\begin{tabular}{lcccccccc}
\hline$a / h$ & 4 & $E r r$. & 10 & \multicolumn{7}{c}{$E r r}$. & 100 & $E r r$. & 1000 & $E r r$. \\
\hline & & & & $F C S R=10$ & & & \\
$3 D$ & 1024.0 & $\%$ & 2369.0 & $\%$ & 15431 & $\%$ & 74092 & $\%$ \\
$C L T$ & 1085.6 & $(6.01)$ & 2713.9 & $(14.5)$ & 27139 & $(75.9)$ & 82782 & $(11.7)$ \\
$F S D T$ & 1085.4 & $(6.00)$ & 2711.8 & $(14.5)$ & 25348 & $(64.3)$ & 79275 & $(7.00)$ \\
$E D 1$ & 1085.4 & $(6.00)$ & 2711.8 & $(14.5)$ & 25348 & $(64.3)$ & 79275 & $(7.00)$ \\
$E D 4$ & 1076.0 & $(5.07)$ & 2581.1 & $(8.95)$ & 16464 & $(6.69)$ & 74801 & $(0.96)$ \\
$E D Z 1$ & 1073.5 & $(4.83)$ & 2555.7 & $(7.88)$ & 16568 & $(7.37)$ & 73860 & $(0.31)$ \\
$E D Z 3$ & 1075.2 & $(5.00)$ & 2753.4 & $(16.2)$ & 16934 & $(9.74)$ & 74110 & $(0.02)$ \\
& & & & $F C S R=10^{5}$ & & & \\
$3 D$ & 1015.1 & $\%$ & 2309.7 & $\%$ & 5854.5 & $\%$ & 6356.0 & $\%$ \\
$C L T$ & 1085.6 & $(6.94)$ & 2714.0 & $(17.5)$ & 27139 & $(>100)$ & 92802 & $(>100)$ \\
$F S D T$ & 1085.5 & $(6.93)$ & 2712.3 & $(17.4)$ & 25681 & $(>100)$ & 87970 & $(>100)$ \\
$E D 1$ & 1085.5 & $(6.93)$ & 2712.3 & $(17.4)$ & 25681 & $(>100)$ & 87970 & $(>100)$ \\
$E D 4$ & 1061.2 & $(4.54)$ & 2410.6 & $(4.37)$ & 6146.6 & $(4.98)$ & 45512 & $(>100)$ \\
$E D Z 1$ & 1061.0 & $(4.52)$ & 2407.3 & $(4.22)$ & 5873.0 & $(0.31)$ & 6357.3 & $(0.02)$ \\
$E D Z 3$ & 1061.3 & $(4.55)$ & 2410.0 & $(4.34)$ & 6101.0 & $(4.20)$ & 7319.5 & $(0.02)$ \\
\hline
\end{tabular}

Table 4

Comparison of various theories to evaluate the fundamental frequency amplitude for the shell with Ren's geometry. $m=0$ and $n=1 . \bar{\omega}=\omega \sqrt{\frac{R_{\beta}^{4}(\rho)_{\text {skin }}}{(E)_{\text {skin }}}}$

\begin{tabular}{lcccccccc}
\hline$R_{\beta} / h$ & 4 & $E r r$. & 10 & $E r r$. & 100 & $E r r$. & 1000 & $E r r$. \\
\hline & & & & $F C S R=10$ \\
$3 D$ & 2.4318 & $\%$ & 3.0453 & $\%$ & 3.2308 & $\%$ & 3.2280 & $\%$ \\
$C L T$ & 3.1915 & $(31.2)$ & 3.2258 & $(5.93)$ & 3.2328 & $(0.06)$ & 3.2329 & $(0.15)$ \\
$F S D T$ & 2.8586 & $(17.5)$ & 3.1588 & $(3.73)$ & 3.2321 & $(0.04)$ & 3.2329 & $(0.15)$ \\
$E D 1$ & 2.8570 & $(17.5)$ & 3.1582 & $(3.71)$ & 3.2321 & $(0.04)$ & 3.2329 & $(0.15)$ \\
$E D 4$ & 2.4891 & $(2.36)$ & 3.0625 & $(0.56)$ & 3.2310 & $(0.01)$ & 3.2329 & $(0.15)$ \\
$E D Z 1$ & 2.4368 & $(0.20)$ & 3.0414 & $(0.13)$ & 3.2308 & $(0.00)$ & 3.2329 & $(0.15)$ \\
$E D Z 3$ & 2.4443 & $(0.51)$ & 3.0471 & $(0.06)$ & 3.2308 & $(0.00)$ & 3.2329 & $(0.15)$ \\
& & & & $F C S R=105$ & & & \\
$3 D$ & 0.2329 & $\%$ & 0.2585 & $\%$ & 1.0654 & $\%$ & 3.4480 & $\%$ \\
$C L T$ & 3.5651 & $(>100)$ & 3.6265 & $(>100)$ & 3.6389 & $(>100)$ & 3.6390 & $(5.53)$ \\
$F S D T$ & 3.1251 & $(>100)$ & 3.5323 & $(>100)$ & 3.6379 & $(>100)$ & 3.6390 & $(5.53)$ \\
$E D 1$ & 3.1233 & $(>100)$ & 3.5316 & $(>100)$ & 3.6379 & $(>100)$ & 3.6390 & $(5.53)$ \\
$E D 4$ & 1.1397 & $(>100)$ & 2.2757 & $(>100)$ & 3.6106 & $(>100)$ & 3.6387 & $(5.53)$ \\
$E D Z 1$ & 0.2431 & $(4.38)$ & 0.2767 & $(7.04)$ & 1.2172 & $(14.2)$ & 3.4526 & $(0.13)$ \\
$E D Z 3$ & 0.2776 & $(19.2)$ & 0.2983 & $(15.4)$ & 1.0742 & $(0.82)$ & 3.4516 & $(0.10)$ \\
\hline
\end{tabular}

means the values $F C S R=10^{1}, 10^{2}, 10^{3}, 10^{4}, 10^{5}$. Results in tables are given only for the two extreme cases which emulate the cases of hard core and very soft core, respectively.

Plate vibration problems are considered in Tables 2 and 3. These refer to fundamental mode $m=n=1$ ( $m, n$ are the wave numbers in the two plate directions) and to higher modes $m=n=100$. Table 2 refers to fundamental frequency parameter of square plates. Soft and very soft core cases (FCSR parameter) are compared for various values of plate thickness parameter $a / h$. The convenience of using ZZ function is evident mainly for the two following reasons: 1. it leads to an error almost independent by FCSR; 2. EDZ1 and EDZ3 are more efficient than ED1 and ED4 in both thick and thin sandwich cases (the error is reduced from $11.1 \%$ to $0.10 \%$ ). The convenience of using ZZF is more evident in the higher order modes case of Table 3 (errors larger than $100 \%$ are reduced to .002\%). In some cases the EDZ1 theory appears better than the EDZ3 one, because of the use of reduced elastic coefficients as suggested in $[9,10]$.

Ren shell geometries [21] are considered in Tables 4 and 5. The following inputs are used: $a=1, b=10.471963$, $\frac{1}{R_{\alpha}}=0, \frac{1}{R_{\beta}}=0.1$ and $\frac{b}{R_{\beta}}=\frac{\pi}{3}$. The conclusions given for plates are confirmed in both cases of fundamental and 
Table 5

Comparison of various theories to evaluate the fundamental frequency amplitude for the shell with Ren's geometry. $m=0$ and $n=100 . \bar{\omega}=\omega \sqrt{\frac{R_{\beta}^{4}(\rho)_{\text {skin }}}{(E)_{\text {skin }} h^{2}}}$

\begin{tabular}{lcccccccc}
\hline$R_{\beta} / h$ & 4 & $E r r$. & 10 & Err. & 100 & Err. & 1000 & $E r r$. \\
\hline & & & & \multicolumn{7}{c}{$F C S R=10$} \\
$3 D$ & 602.89 & $\%$ & 1404.2 & $\%$ & 10385 & $\%$ & 36021 & $\%$ \\
$C L T$ & 743.33 & $(23.3)$ & 1836.7 & $(30.8)$ & 18325 & $(76.4)$ & 38063 & $(5.67)$ \\
$F S D T$ & 667.62 & $(10.7)$ & 1765.6 & $(25.7)$ & 16037 & $(54.4)$ & 37278 & $(3.49)$ \\
$E D 1$ & 667.62 & $(10.7)$ & 1765.6 & $(25.7)$ & 16037 & $(54.4)$ & 37278 & $(3.49)$ \\
$E D 4$ & 648.68 & $(7.59)$ & 1589.7 & $(13.2)$ & 10942 & $(5.36)$ & 36200 & $(0.50)$ \\
$E D Z 1$ & 660.58 & $(9.57)$ & 1623.4 & $(15.6)$ & 10665 & $(2.70)$ & 35961 & $(0.16)$ \\
$E D Z 3$ & 653.64 & $(8.41)$ & 1628.1 & $(15.9)$ & 10874 & $(4.71)$ & 36023 & $(0.00)$ \\
& & & & $F C S R=10^{5}$ & & & \\
$3 D$ & 593.50 & $\%$ & 1338.9 & $\%$ & 2698.1 & $\%$ & 3053.6 & $\%$ \\
$C L T$ & 746.90 & $(25.8)$ & 1838.1 & $(37.3)$ & 18326 & $(>100)$ & 42763 & $(>100)$ \\
$F S D T$ & 659.01 & $(11.0)$ & 1756.1 & $(31.1)$ & 16421 & $(>100)$ & 41662 & $(>100)$ \\
$E D 1$ & 659.01 & $(11.0)$ & 1756.1 & $(31.1)$ & 16421 & $(>100)$ & 41662 & $(>100)$ \\
$E D 4$ & 627.17 & $(5.67)$ & 1415.7 & $(5.74)$ & 4333.8 & $(>100)$ & 27134 & $(>100)$ \\
$E D Z 1$ & 643.54 & $(8.43)$ & 1459.2 & $(8.98)$ & 2725.1 & $(1.00)$ & 3054.2 & $(0.02)$ \\
$E D Z 3$ & 627.90 & $(5.80)$ & 1418.4 & $(5.94)$ & 2954.4 & $(9.50)$ & 3473.2 & $(13.7)$ \\
\hline
\end{tabular}

Table 6

Comparison of various theories to evaluate the fundamental frequency amplitude for the shell with Bhaskar and Varadan's geometry. $m=1$ and $n=8 \cdot \bar{\omega}=\omega \sqrt{\frac{R_{\beta}^{4}(\rho)_{\text {skin }}}{(E)_{\text {skin }} h^{2}}}$

\begin{tabular}{lcccccccc}
\hline$R_{\beta} / h$ & 4 & $E r r$. & 10 & Err. & 100 & Err. & 1000 & Err. \\
\hline & & & & \multicolumn{2}{c}{$F C S R=10$} \\
$3 D$ & 4.1315 & $\%$ & 5.7896 & $\%$ & 7.3593 & $\%$ & 36.527 & $\%$ \\
$C L T$ & 6.1222 & $(48.2)$ & 6.3897 & $(10.4)$ & 7.3700 & $(0.14)$ & 36.528 & $(0.00)$ \\
$F S D T$ & 5.1639 & $(25.0)$ & 6.1564 & $(6.33)$ & 7.3677 & $(0.11)$ & 36.528 & $(0.00)$ \\
$E D 1$ & 5.1610 & $(24.9)$ & 6.1501 & $(6.23)$ & 7.1174 & $(3.29)$ & 31.233 & $(14.5)$ \\
$E D 4$ & 4.2581 & $(3.06)$ & 5.8415 & $(0.90)$ & 7.3599 & $(0.01)$ & 36.527 & $(0.00)$ \\
$E D Z 1$ & 4.1454 & $(0.34)$ & 5.7761 & $(0.23)$ & 7.1130 & $(3.35)$ & 31.233 & $(14.5)$ \\
$E D Z 3$ & 4.1626 & $(0.75)$ & 5.7937 & $(0.07)$ & 7.3593 & $(0.00)$ & 36.527 & $(0.00)$ \\
& & & & $F C S R=105$ & & & \\
$3 D$ & 0.4478 & $\%$ & 0.6105 & $\%$ & 3.9470 & $\%$ & 36.557 & $\%$ \\
$C L T$ & 6.6779 & $(>100)$ & 7.1693 & $(>100)$ & 8.0849 & $(>100)$ & 36.679 & $(0.33)$ \\
$F S D T$ & 5.5626 & $(>100)$ & 6.8458 & $(>100)$ & 8.0816 & $(>100)$ & 36.678 & $(0.33)$ \\
$E D 1$ & 5.5602 & $(>100)$ & 6.8394 & $(>100)$ & 7.8584 & $(>100)$ & 31.409 & $(14.1)$ \\
$E D 4$ & 1.7482 & $(>100)$ & 3.7193 & $(>100)$ & 7.9891 & $(>100)$ & 36.677 & $(0.33)$ \\
$E D Z 1$ & 0.5318 & $(18.7)$ & 0.6228 & $(2.01)$ & 3.5481 & $(10.1)$ & 31.269 & $(14.5)$ \\
$E D Z 3$ & 0.5459 & $(21.9)$ & 0.6742 & $(10.4)$ & 3.9565 & $(0.24)$ & 36.557 & $(0.00)$ \\
\hline
\end{tabular}

higher in-plane vibration modes. Similar percentage errors of plate cases have been found. The vibration response of cylindrical shell by Bhaskar and Varadan [22] is analyzed in Tables 6 and 7. The following inputs are used: $a=40, b=62.831853, \frac{a}{R_{\beta}}=4, \frac{1}{R_{\alpha}}=0$ and $\frac{1}{R_{\beta}}=0.1$. It is concluded that the benefits (already found in the plate analysis) of using ZZF are fully confirmed for shell geometries.

\section{Conclusions}

The present paper has demonstrated the convenience of using the Zig-Zag function to build higher order theories to evaluate the free vibration response of sandwich plates and shells. Soft and very soft core cases have been considered along with different values of plate/shell geometrical parameters. Frequency parameters related to fundamental and higher vibration modes are compared. The conducted numerical investigation has shown that very significant improvements are obtained by using the ZZF for both fundamental and higher vibration modes of sandwich plates and shells. The use of EDZ1 theory is highly recommended for thin sandwich structures with soft core. Such 
Table 7

Comparison of various theories to evaluate the fundamental frequency amplitude for the shell with Bhaskar and Varadan's geometry. $m=1$ and $n=100 . \bar{\omega}=\omega \sqrt{\frac{R_{\beta}^{4}(\rho)_{s k i n}}{(E)_{s k i n} h^{2}}}$

\begin{tabular}{|c|c|c|c|c|c|c|c|c|}
\hline$R_{\beta} / h$ & 4 & Err. & 10 & Err. & 100 & Err. & 1000 & Err. \\
\hline & \multicolumn{8}{|c|}{$F C S R=10$} \\
\hline $3 D$ & 66.767 & $\%$ & 171.50 & $\%$ & 911.81 & $\%$ & 1062.6 & $\%$ \\
\hline$C L T$ & 123.92 & (85.6) & 306.15 & (78.5) & 1043.9 & (14.5) & 1064.3 & $(0.03)$ \\
\hline$F S D T$ & 112.48 & (68.5) & 289.56 & (68.8) & 989.52 & $(8.52)$ & 1063.7 & $(0.10)$ \\
\hline$E D 1$ & 112.48 & (68.5) & 288.62 & (68.3) & 989.52 & $(8.52)$ & 1063.7 & $(0.10)$ \\
\hline$E D 4$ & 79.158 & (18.5) & 184.86 & (7.79) & 922.28 & (1.15) & 1062.7 & $(0.01)$ \\
\hline$E D Z 1$ & 89.193 & (33.6) & 189.33 & (10.4) & 908.47 & $(0.37)$ & 1062.5 & $(0.01)$ \\
\hline$E D Z 3$ & 88.189 & (33.6) & 192.06 & (12.0) & 912.16 & $(0.04)$ & 1062.6 & $(0.00)$ \\
\hline \multicolumn{9}{|c|}{$F C S R=10^{5}$} \\
\hline $3 D$ & 51.687 & $\%$ & 67.519 & $\%$ & 79.682 & $\%$ & 227.04 & $\%$ \\
\hline$C L T$ & 124.53 & $(>100)$ & 306.39 & $(>100)$ & 1169.0 & $(>100)$ & 1198.0 & $(>100)$ \\
\hline$F S D T$ & 110.85 & $(>100)$ & 292.88 & $(>100)$ & 1094.7 & $(>100)$ & 1197.1 & $(>100)$ \\
\hline$E D 1$ & 110.84 & $(>100)$ & 291.22 & $(>100)$ & 1094.7 & $(>100)$ & 1197.1 & $(>100)$ \\
\hline$E D 4$ & 55.126 & $(6.65)$ & 73.022 & $(8.15)$ & 525.69 & $(>100)$ & 1173.3 & $(>100)$ \\
\hline$E D Z 1$ & 62.846 & (21.6) & 73.876 & $(9.41)$ & 79.705 & $(0.03)$ & 227.22 & $(0.08)$ \\
\hline$E D Z 3$ & 55.267 & (6.93) & 72.746 & (7.74) & 91.989 & (15.4) & 231.37 & (1.91) \\
\hline
\end{tabular}

improvements are almost independent by the face-to-core stiffness ratio; this is not if ZZF is discarded by the considered sandwich theory. What above confirms the conclusions already drown in recent papers devoted to the bending analysis of sandwich plates [3]. The proposed results show the effectiveness and benefits of using ZZF in the free vibration analysis of sandwich structures. The simplicity of its implementation makes it very attractive. The main limitation of the ZZF appears in case of very soft cores and moderately thick sandwiches, in particular to evaluate the transverse displacements and the transverse shear/normal stresses, in this case the use of Layer Wise theory is recommended with respect to Equivalent Single Layers ones including or not the ZZF.

\section{References}

[1] H. Altenbach, Theories for laminated and sandwich plates. A review, International Applied Mechanics 34 (1998), 243-252.

[2] T.N. Bitzer, Honeycomb Technology, Chapman and Hall, London, 1997.

[3] S. Brischetto, E. Carrera and L. Demasi, Improved bending analysis of sandwich plate by using Zig-Zag function, Composite Structures, in press.

[4] S. Burton and A.K. Noor, Assessment of computational model for sandwich panels and shells, Computer Methods in Applied Mechanics and Engineering 124 (1995), 125-151.

[5] E. Carrera, A historical review of zig-zag theories for multilayered plates and shells, Applied Mechanics Reviews 56 (2003), $290-309$.

[6] E. Carrera, On the use of Murakami's Zig-Zag function in the modeling of layered plates and shells, Composite Structures 82 (2004), 541-554.

[7] E. Carrera, Theories and Finite Elements for multilayered plates and shells: a unified compact formulation with numerical assessment and benchmarking, Archives of Computational Methods in Engineering, State of the Art Reviews 10 (2003), 215-296.

[8] E. Carrera and S. Brischetto, A survey with numerical assessment of classical and refined theories for the analysis of sandwich plates, Applied Mechanics Reviews, in press.

[9] E. Carrera and S. Brischetto, Analysis of thickness locking in classical, refined and mixed multilayered plate theories, Composite Structures 82 (2008), 549-562.

[10] E. Carrera and S. Brischetto, Analysis of thickness locking in classical, refined and mixed theories for layered shells, Composite Structures 85 (2008), 83-90.

[11] E. Carrera, S. Brischetto and A. Robaldo, Variable kinematic model for the analysis of functionally graded material plates, AIAA Journal 46 (2008), 194-203.

[12] L. Demasi, Refined multilayered plate elements based on Murakami Zig-Zag function, Computers \& Structures 70 (2005), $308-316$.

[13] L. Demasi, 2D, quasi 3D and 3D exact solutions for bending of thick and thin sandwich plates, Journal of Sandwich Structures and Materials 10 (2008), 271-310.

[14] L. Demasi, $\infty^{3}$ Hierarchy plate theories for thick and thin composite plates: the generalized unified formulation, Composite Structures 84 (2008), 256-270.

[15] G. Kirchhoff, Uber das Gleichgewicht und die Bewegung einer elastischen Scheibe, J Angew Math 40 (1850), 51-88.

[16] A.W. Leissa, Vibration of Shells, NASA SP-288, National Aeronautics and Space Administration, Washington, D.C., 1973.

[17] L. Librescu and T. Hause, Recent developments in the modeling and behaviors of advanced sandwich constructions: a survey, Composite Structures 48 (2000), 1-17. 
[18] H. Murakami, Laminated composite plate theory with improved in-plane responses, Journal of Applied Mechanics 53 (1986), $661-666$.

[19] A.K. Noor, S. Burton and C.W. Bert, Computational model for sandwich panels and shells, Applied Mechanics Reviews 49 (1996), $155-199$.

[20] E. Reissner, The effect of transverse shear deformation on the bending of elastic plates, Journal of Applied Mechanics 12 (1945), 69-76.

[21] J.G. Ren, Exact solutions for laminated cylindrical shells in cylindrical bending, Composite Science and Technology 29 (1987), $169-187$.

[22] T.K. Varadan and K. Bhaskar, Bending of laminated orthotropic cylindrical shells, Composite Structures 17 (1991), $141-156$.

[23] J.R. Vinson, Sandwich structures, Applied Mechanics Reviews 54 (2001), 201-214.

[24] J.R. Vinson, The Behavior of Sandwich Structures of Isotropic and Composite Materials, Technomic Publishing Co., Lancaster, PA, 1999.

[25] D. Zenkert, An Introduction to Sandwich Structures, Chamelon Press, Oxford, 1995. 

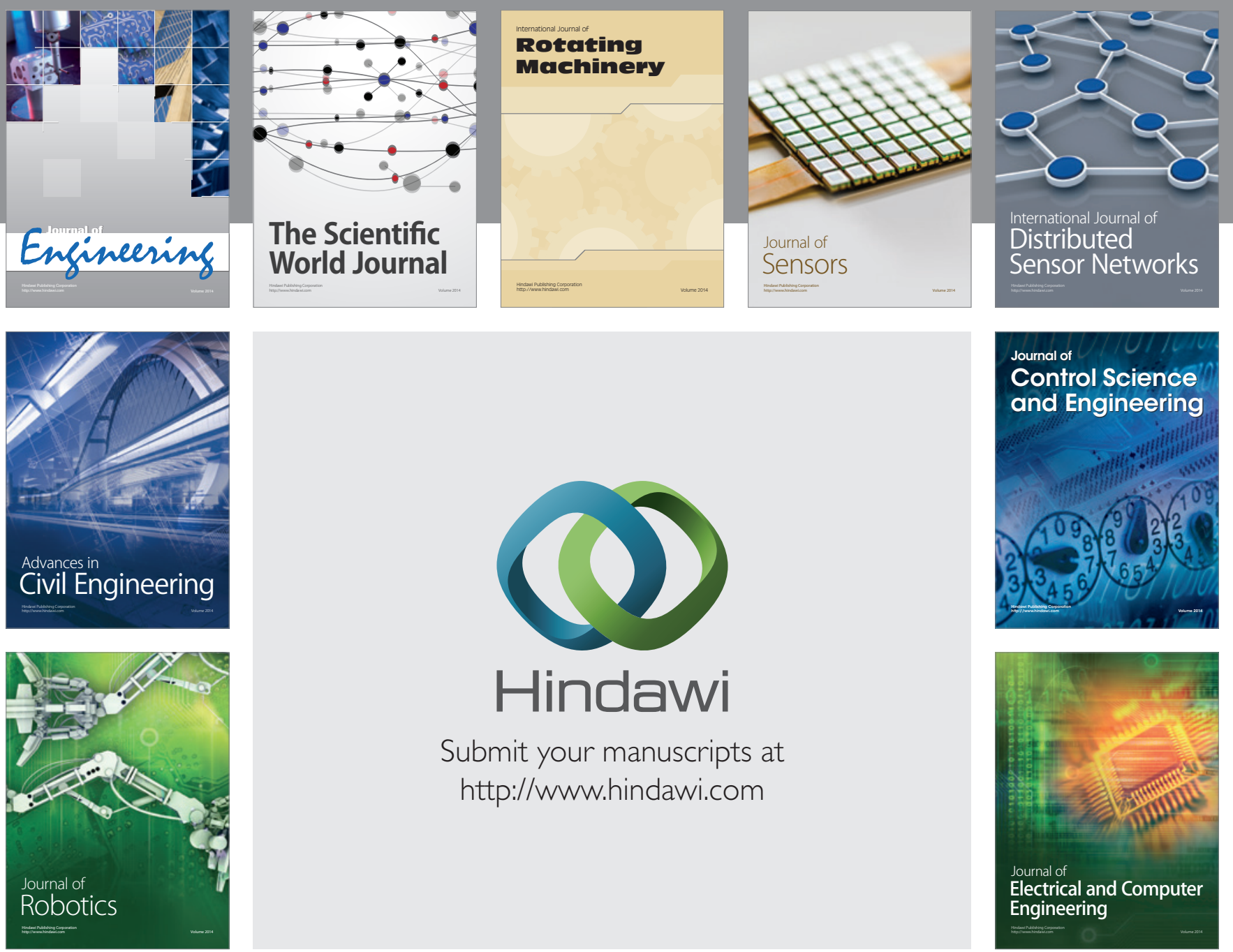

Submit your manuscripts at

http://www.hindawi.com
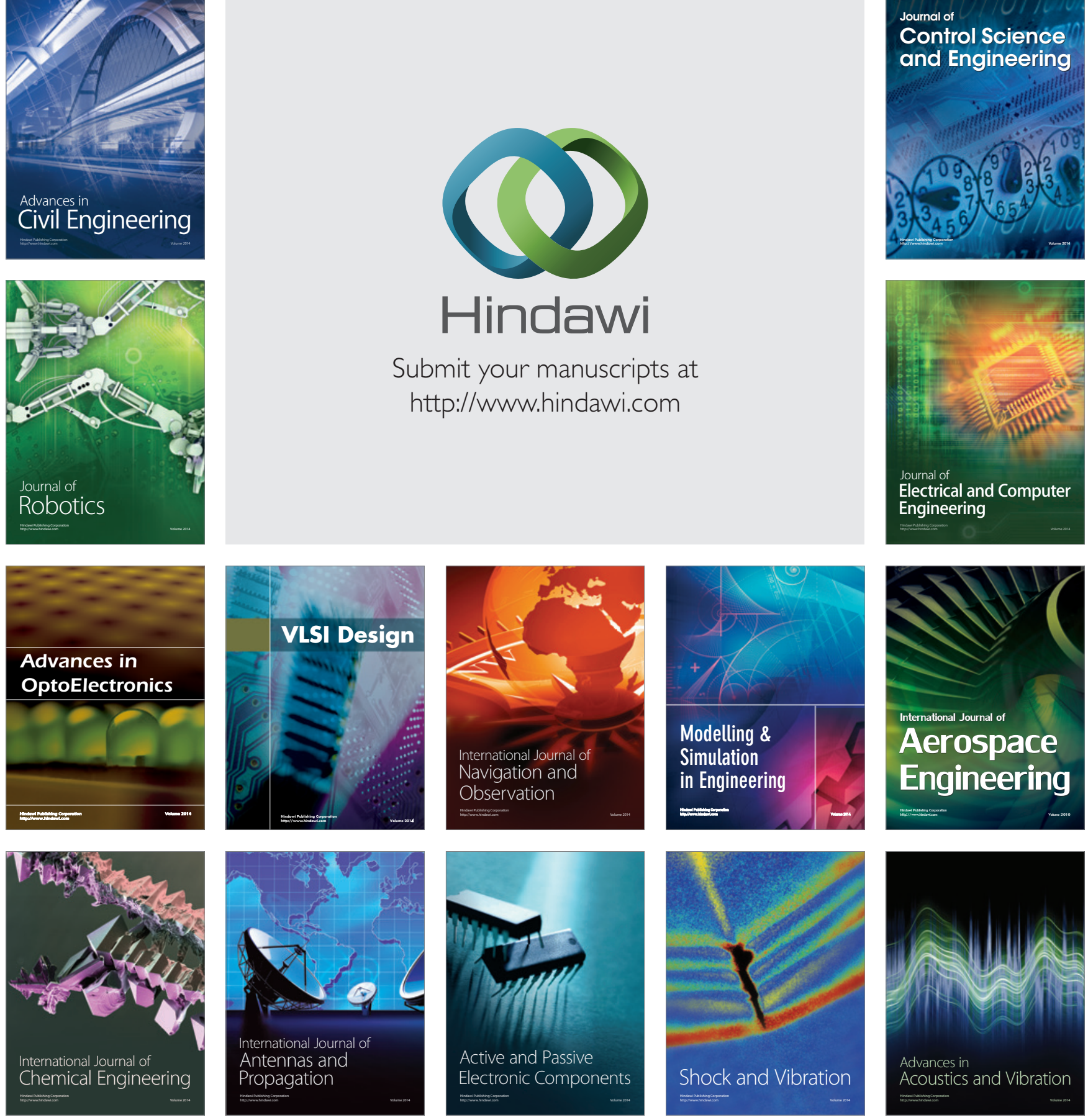